\title{
DIAGNÓSTICOS DE ENFERMAGEM PREVALENTES EM PACIENTES INTERNADOS NA UNIDADE DE TERAPIA INTENSIVA: REVISÃO INTEGRATIVA
}

\section{PREVALENT NURSING DIAGNOSIS IN PATIENTS HOSPITALIZED IN INTENSIVE CARE UNIT: AN INTEGRATIVE REVIEW}

\author{
Rudval Souza da Silva*, Marylia de Oliveira Mendes Lima**, Willian Cássia de Oliveira \\ Bandeira**, Agnete Troelsen Pereira***, Antônia Adônis Callou Sampaio****, Gilvânia Patrícia \\ do Nascimento Paixão*****
}

\begin{abstract}
Autor para correspondência: Rudval Souza da Silva - rudvalsouza@yahoo.com.br
*Professor Adjunto da Universidade do Estado da Bahia (UNEB - Senhor do Bonfim). Doutor em Enfermagem pela Universidade Federal da Bahia (UFBA). Especialista em Cuidados Paliativos pela Asociación Pallium Latinoamérica Universidad Del Salvador, Buenos Aires, Argentina.

**Enfermeira com pós-graduação em Enfermagem em Emergência e Terapia Intensiva pela Universidade do Estado da Bahia (UNEB - Senhor do Bonfim).

***Professora Auxiliar da Universidade do Estado da Bahia (UNEB - Senhor do Bonfim).

****Mestre em Gestão pela UNEB. Professora Auxiliar da Universidade do Estado da Bahia (UNEB - Senhor do Bonfim).

******Professora Assistente da Universidade do Estado da Bahia (UNEB - Senhor do Bonfim). Mestre e Doutoranda em

Enfermagem pela UFBA.
\end{abstract}

\section{R E S U M O}

diagnóstico de enfermagem é resultado de um processo de interpretação e agrupamento das queixas dos pacientes a partir do julgamento clínico dos dados coletados na etapa de investigação, direcionado pelo pensamento crítico do enfermeiro. Objetivo: identificar a prevalência dos diagnósticos de enfermagem nos pacientes assistidos na UTI. Método: revisão integrativa de caráter exploratório e descritivo. Foi realizada a identificação de estudos através de levantamento bibliográfico nas bases de dados: LILACS e SciELO resultando numa amostra final de 11 estudos que compuseram o corpus da pesquisa. Resultados: dos 11 estudos analisados foram identificados 91 diagnósticos de enfermagem. Estabeleceu-se um Índice de Concordância $(\geq 0,60)$ para a análise e foram considerados como prevalentes apenas os diagnósticos de enfermagem que alcançaram $\circ \mathrm{IC}$, ○ que resultou num total de 10 diagnósticos de enfermagem mais prevalentes: risco de infecção, volume de líquido excessivo, risco de aspiração, risco de integridade da pele prejudicada, integridade da pele prejudicada, mobilidade física prejudicada, débito cardíaco diminuído, déficit de autocuidado higiene íntima, padrão respiratório ineficaz e desobstrução ineficaz das vias áreas. Conclusão: foi possível evidenciar que no contexto da terapia intensiva são mais prevalentes os diagnósticos reais do que os de riscos. Com a identificação da prevalência dos diagnósticos de enfermagem foi possível traçar o perfil clínico e epidemiológico dos pacientes internados numa UTI.

Palavras chaves: Processos de Enfermagem; Diagnóstico de Enfermagem; Unidades de Terapia Intensiva. 
The nursing diagnosis is the result of a process of gathering and interpreting the complaints from patients employing clinical judgment of data collected in the investigatory step, directing the critical thinking by the nurses. Objective: to identify the prevalence of nursing diagnoses in assisted patients in the ICU. Method: it's an integrative review, of exploratory and descriptive nature and the data were collected in the databases LILACS and SciELO resulting in a final sample of 11 studies that made up the corpus of the research. Results: those 11 studies were analyzed and in them 91 nursing diagnoses have been identified. A Concordance Index (IC $\geq 0.60$ ) for analysis was established and only prevalent nursing diagnoses that have achieved IC have been taken in consideration for analysis, resulting in a total of 10 prevalent nursing diagnoses: risk of infection, excessive fluid volume, risk of aspiration, impaired skin integrity risk, impaired skin integrity, impaired physical mobility, decreased cardiac output, intimate hygiene and self-care deficit, ineffective breathing pattern and ineffective clearance of the airways. Conclusion: it was possible to observe that in the context of intensive care are more prevalent the actual diagnosis than those of the risks. With the identification of the prevalence of nursing diagnoses it was possible to trace the clinical and epidemiological profile of patients admitted to an ICU.

Keywords: Nursing Process; Nursing Diagnostic; Intensive Care Unit. 


\section{INTRODUÇÃO}

A Unidade de Terapia Intensiva (UTI) é um ambiente hospitalar que se destina à prestação de cuidados a pessoa em condições críticas, recuperável e requer um atendimento contínuo, especializado e humano'.

A sistematização do trabalho desenvolvido pela equipe de enfermagem deve atender as necessidades de um cuidado especializado e complexo conforme requer o perfil da UTI, em prol de uma assistência de qualidade, com eficiência e eficácia ${ }^{2}$. A elevada procura por esses serviços, o quantitativo de procedimentos realizados nesse ambiente e o número reduzido de vagas favorecem - repensar sobre a necessidade de organização das ações executadas e do seu acompanhamento. Assim, a rotina da assistência de enfermagem aos pacientes na UTI necessita de um redirecionamento dos cuidados, objetivando a integralidade e individualidade da assistência prestada ${ }^{3}$.

Para a organização das práticas de cuidar, fazse necessária à implantação e implementação da Sistematização da Assistência de Enfermagem (SAE) como uma ferramenta que visa organiza o trabalho profissional da equipe de enfermagem quanto ao método, pessoal e instrumentos, tornando possível a operacionalização do Processo de Enfermagem, enquanto dinâmica de ações sistematizadas e interrelacionadas com a finalidade de prestar assistência de qualidade a uma clientela (pessoa, família ou comunidade) $)^{4}$.

Dessa forma, a implementação da SAE e do PE tornam-se requisitos fundamentais nos serviços de enfermagem, e mais especificamente na UTI que é o cerne das discussões neste estudo, em prol da uniformização do trabalho da equipe de enfermagem, evitando a fragmentação dos cuidados e possibilitando um elo de comunicação entre todos os profissionais de saúde, estabelecendo assim, uma comunicação clara e objetiva'.

O Processo de Enfermagem é desenvolvido em cinco etapas inter-relacionadas: coleta de dados, que consiste no levantamento do histórico pessoal e nosológico do paciente; diagnóstico de enfermagem que direciona $\circ$ pensamento crítico do enfermeiro na descrição do quadro clínico do indivíduo; planejamento, o qual direcionará as ações/intervenções de enfermagem para o cuidado da uma clientela (pessoa, família ou comunidade); implementação, concerne a aplicação das intervenções de enfermagem; e avaliação de enfermagem que culmina na análise quanto ao alcance ou não dos resultados esperados ${ }^{4-6}$.

Considerando a relevância da etapa de diagnóstico de enfermagem no direcionamento do pensamento crítico e julgamento clínico do enfermeiro, a presente pesquisa se atentará a esta etapa do $P E$, a qual ocorre por meio de um processo de interpretação e agrupamento das queixas dos pacientes a partir do julgamento clínico dos dados coletados na primeira etapa que servem como base norteadora para a seleção das intervenções necessárias ao alcance dos resultados esperados ${ }^{4}$.

Parte-se do pressuposto de que a identificação de um conjunto de diagnósticos de enfermagem prevalentes no contexto da terapia intensiva pode contribuir para o estabelecimento de um perfil diagnóstico a ser adotado na assistência aos pacientes internados nesse serviço, levando em consideração o referencial teórico da Enfermagem. A partir dos diagnósticos mais frequente nos pacientes internados na UTI, será possível direcionar a assistência de enfermagem, subsidiando o enfermeiro na elaboração de um plano de cuidado.

Destaca-se também que, com o uso dos diagnósticos de enfermagem, espera-se possibilitar uma maior visibilidade das ações da equipe de enfermagem para além das habilidades técnicas, direcionando um cuidar embasado no raciocínio clínico e no conhecimento científico - uma Enfermagem Baseada em Evidências.

Nesse contexto, o presente artigo buscou responder a seguinte questão de pesquisa: quais os diagnósticos de enfermagem mais prevalentes nos pacientes assistidos na UTI? Assim, define-se como objetivo identificar a prevalência dos diagnósticos de enfermagem nos pacientes assistidos na UTI. 


\section{MÉTODO}

Trata-se de uma revisão integrativa de caráter exploratório e descritivo, mediante a realização das seis etapas inerentes ao método: definição da questão norteadora e objetivos da pesquisa; delimitação das buscas através dos critérios de inclusão e exclusão; sumarização das informações colhidas; análise crítica dos achados; interpretação dos resultados; e descrição do trabalho ${ }^{7-8}$.

Inicialmente realizou-se a identificação de estudos através de levantamento bibliográfico nas bases de dados: LILACS (Literatura Latino- Americana e do Caribe) e SciELO (Scientific Eletronic Library Online), utilizando os seguintes descritores controlados: "diagnóstico de enfermagem" e "unidade de terapia intensiva". Deve-se ressaltar a utilização do recurso booleano "and", associando os dois descritores. As buscas foram realizadas no mês de abril de 2016.

A delimitação da pesquisa ocorreu através dos seguintes critérios de inclusão: trabalhos científicos publicados em periódicos de consulta livre; que contenha em seus títulos e/ou resumos os seguintes descritores: diagnóstico de enfermagem e unidade de terapia intensiva; disponibilizados na íntegra e publicados no idioma português com recorte temporal entre os anos de 2002 a 2015. Como critério de exclusão: estudos que não tratem dos diagnósticos de enfermagem na UTI; textos publicados em outros idiomas que não na língua portuguesa; e não disponíveis na íntegra.

Deve-se ressaltar que o período de 2002 a 2015 foi determinado em razão da Sistematização da Assistência de Enfermagem tornar-se normatizada pelo Conselho Federal de Enfermagem pela primeira vez em 2002, com a publicação da Resolução COFEN $n^{\circ} 272 / 2002^{9}$ tornando-a obrigatória e consequentemente, sendo implantada com maior vigor em todos os ambientes de saúde, inclusive na UTI.
Objetivando identificar os diagnósticos de enfermagem mais prevalentes dentre aqueles 91 levantados nos estudos, estes foram digitados numa planilha no Software Microsoft Office Excel ${ }^{\circledR}$ 2010 e analisados estatisticamente a partir do estabelecimento de um Índice de Concordância (IC $\geq 0,60$ ) de modo que foram considerados como prevalentes apenas os diagnósticos de enfermagem que alcançaram este IC, o qual indica que no mínimo em $60 \%$ dos estudos analisados foi evidenciando sua prevalência.

Quanto às questões éticas, foram respeitados os princípios da honestidade e fidedignidade, bem como os direitos autorais $\mathrm{e}$, em virtude da natureza bibliográfica da pesquisa, não houve necessidade de aprovação no Comitê de Ética em Pesquisa (CEP).

\section{RESULTADOS}

Em suma, identificaram-se 131 artigos entre as duas bases de dados com a aplicação dos dois descritores. Após a aplicação dos critérios de inclusão e exclusão e uma leitura dos títulos e resumos esse quantitativo foi reduzido a 24 publicações, as quais foram selecionadas e passaram por uma leitura detalhadas e analítica em busca de responder ao objetivo do estudo. Logo, alguns manuscritos foram excluídos por não atender ao objetivo do estudo e resultou numa amostra final de 11 artigos que constitui o corpus da pesquisa. Na Figura 01 apresenta-se detalhadamente o percurso metodológico seguido para a seleção dos estudos a compor o corpus da pesquisa. 


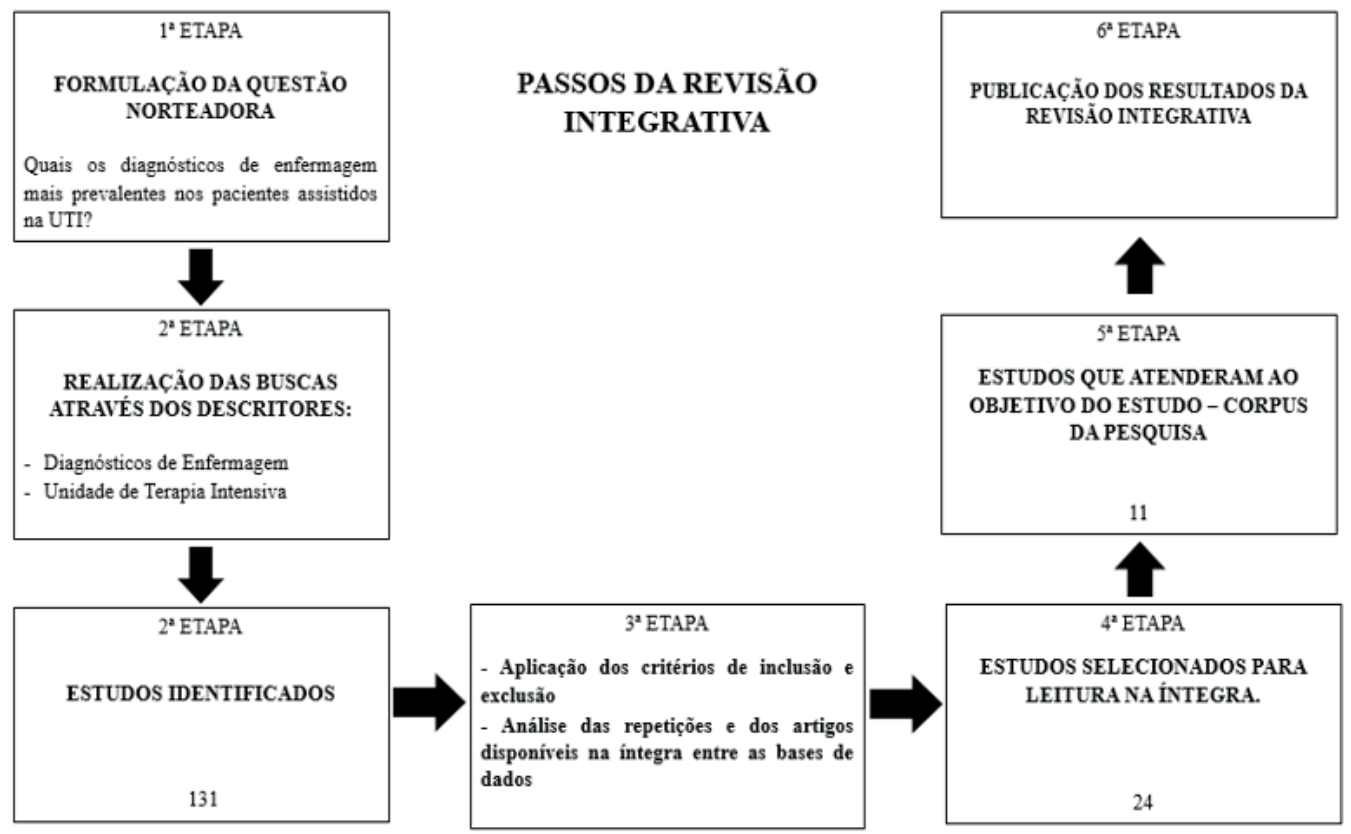

Figura 01: Fluxograma da seleção amostral dos estudos incluídos na Revisão Integrativa. Senhor do Bonfim, BA, Brasil, 2016.

Apresenta-se no quadro a seguir (Quadro 01) o corpus da pesquisa com as seguintes variáveis: autor, ano, base de dados consultada, título, tipo de estudo e objetivo.

Quadro 01: Distribuição dos estudos segundo autor/ano, base de dados consultada, título, tipo de estudo e objetivo. Senhor do Bonfim, BA, Brasil, 2016.

\begin{tabular}{|c|c|c|c|c|}
\hline ESTUDOS & $\begin{array}{l}\text { AUTOR/ANO } \\
\text { BASE DE DADOS }\end{array}$ & TíTULO & TIPO DE ESTUDO & OBJETIVO \\
\hline E-1 & $\begin{array}{l}\text { CARVALHO } \\
\text { et al. } / 2008 \\
\text { LILACS }\end{array}$ & $\begin{array}{l}\text { Relações entre coleta de dados, } \\
\text { diagnósticos e prescrições de } \\
\text { enfermagem a pacientes adultos de } \\
\text { uma Unidade de Terapia Intensiva. }\end{array}$ & $\begin{array}{l}\text { Estudo descritivo de } \\
\text { caráter retrospectivo }\end{array}$ & $\begin{array}{c}\text { Analisar as relações entre a coleta de dados, o estabe- } \\
\text { lecimento de diagnósticos e elaboração de prescriçães } \\
\text { de enfermagem a adultos internados em uma unidade de } \\
\text { terapia intensiva. }\end{array}$ \\
\hline $\mathrm{E}-2$ & $\begin{array}{l}\text { OLIVEIRA; } \\
\text { FREITAS } / 2009 \\
\text { LILACS }\end{array}$ & $\begin{array}{l}\text { Diagnósticos e intervenções de } \\
\text { enfermagem frequentes em mulheres } \\
\text { internadas em uma unidade de } \\
\text { terapia intensiva. }\end{array}$ & $\begin{array}{l}\text { Estudo descritivo e } \\
\text { retrospectivo }\end{array}$ & $\begin{array}{l}\text { Identificar os diagnósticos de enfermagem mais fre- } \\
\text { quentes e propor intervenções para mulheres internadas } \\
\text { em uma unidade de terapia intensiva materna de um } \\
\text { Hospital público de Fortaleza-CE. }\end{array}$ \\
\hline E-3 & $\begin{array}{c}\text { PRADO; } \\
\text { BECCARIA; } \\
\text { CONTRIN/2009 } \\
\text { LILACS }\end{array}$ & $\begin{array}{l}\text { Principais Diagnósticos de Enfermagem } \\
\text { na Unidade de Terapia Intensiva. }\end{array}$ & Revisão bibliográfica & $\begin{array}{l}\text { Identificar os principais diagnósticos de enfermagem, } \\
\text { publicados por estudos desenvolvidos em unidades de } \\
\text { tratamento intensivo. }\end{array}$ \\
\hline E-4 & $\begin{array}{c}\text { TRUPPEL } \\
\text { et al. } / 2009 \\
\text { LILACS }\end{array}$ & $\begin{array}{l}\text { Sistematização da Assistência de } \\
\text { Enfermagem na Unidade de Terapia } \\
\text { Intensiva. }\end{array}$ & Estudo transversal & $\begin{array}{l}\text { Reestruturar a Sistematização da Assistência de Enferma- } \\
\text { gem (SAE) em uma Unidade de Terapia Intensiva (UTI). }\end{array}$ \\
\hline E-5 & $\begin{array}{c}\text { CRUZ, } \\
\text { LOPES/2010 } \\
\text { LILACS }\end{array}$ & $\begin{array}{l}\text { Diagnósticos de Enfermagem no pós- } \\
\text { operatório de cirurgias cardíacas. }\end{array}$ & $\begin{array}{l}\text { Estudo descritivo e } \\
\text { documental }\end{array}$ & $\begin{array}{c}\text { Identificar os principais diagnósticos de enfermagem no } \\
\text { pós-operatório de cirurgia cardíaca em uma unidade } \\
\text { de terapia intensiva de hospital público no município de } \\
\text { Bauru. }\end{array}$ \\
\hline$E-6$ & $\begin{array}{l}\text { PEGANIN } \\
\text { et al. } / 2010 \\
\text { LILACS }\end{array}$ & $\begin{array}{l}\text { Implantação do Diagnóstico de } \\
\text { Enfermagem em Unidade de Terapia } \\
\text { Intensiva: uma análise periódica. }\end{array}$ & Estudo transversal & $\begin{array}{l}\text { Identificar os principais diagnósticos de enfermagem de } \\
\text { uma unidade de terapia intensiva, comparando-se as di- } \\
\text { ferentes categorias entre os pacientes clínicos e cirúrgicos. }\end{array}$ \\
\hline
\end{tabular}


Quadro 01: Distribuição dos estudos segundo autor/ano, base de dados consultada, título, tipo de estudo e objetivo. Senhor do Bonfim, BA, Brasil, 2016.

(continuação)

\begin{tabular}{|c|c|c|c|c|}
\hline ESTUDOS & $\begin{array}{l}\text { AUTOR/ANO } \\
\text { BASE DE DADOS }\end{array}$ & TíTULO & TIPO DE ESTUDO & OBJETIVO \\
\hline $\mathrm{E}-7$ & $\begin{array}{l}\text { SALOMÉ/2011 } \\
\text { LILACS }\end{array}$ & $\begin{array}{l}\text { Diagnósticos de Enfermagem de } \\
\text { pacientes internados na Unidade de } \\
\text { Terapia Intensiva }\end{array}$ & Estudo descritivo & $\begin{array}{c}\text { Identificar os diagnósticos de enfermagem, segundo } \\
\text { a Taxonomia I da North American Nursing Diagnoses } \\
\text { Association - NANDA e os padrões de resposta humana, } \\
\text { apresentados por pacientes internados em uma Unidade } \\
\text { de Terapia }\end{array}$ \\
\hline$E-8$ & $\begin{array}{c}\text { CHIANCA; LIMA; } \\
\text { SALGADO/2012 } \\
\text { SCIELO }\end{array}$ & $\begin{array}{l}\text { Diagnósticos de enfermagem } \\
\text { identificados em pacientes internados } \\
\text { em Unidade de Terapia Intensiva } \\
\text { Adulto }\end{array}$ & Estudo descritivo & $\begin{array}{l}\text { Identificar nos registros de prontuários de pacientes } \\
\text { internados em uma UTI de adultos os diagnósticos de } \\
\text { enfermagem e mapear os diagnósticos mais frequentes } \\
\text { às Necessidades Humanas Básicas de Horta. }\end{array}$ \\
\hline E-9 & $\begin{array}{l}\text { ALMEIDA } \\
\text { et al. } / 2013 \\
\text { SCIELO }\end{array}$ & $\begin{array}{l}\text { Diagnósticos de enfermagem mais } \\
\text { frequentes em pacientes internados } \\
\text { na Unidade de Terapia Intensiva } \\
\text { Coronariana. }\end{array}$ & $\begin{array}{l}\text { Estudo descritivo, } \\
\text { exploratório } \\
\text { de abordagem } \\
\text { quantitativa. }\end{array}$ & $\begin{array}{l}\text { Identificar os diagnósticos de enfermagem da taxonomia } \\
\text { II da NANDA mais frequentes de pacientes internados em } \\
\text { Unidade de Terapia Intensiva Coronariana. }\end{array}$ \\
\hline E-10 & $\begin{array}{c}\text { RAMOS } \\
\text { et al. } / 2013 \\
\text { SCIELO }\end{array}$ & $\begin{array}{c}\text { Diagnósticos de Enfermagem } \\
\text { Documentados em Prontuários de } \\
\text { Pacientes em Unidade de Terapia } \\
\text { Intensiva }\end{array}$ & $\begin{array}{l}\text { Estudo descritivo e } \\
\text { Documental. }\end{array}$ & $\begin{array}{l}\text { Analisar o perfil de diagnósticos de enfermagem em } \\
\text { relação ao tempo de permanência e evolução clínica de } \\
\text { pacientes internados na Unidade de Terapia Intensiva de } \\
\text { um município de Minas Gerais. }\end{array}$ \\
\hline E-1 1 & $\begin{array}{c}\text { GUEDES; } \\
\text { ROSSATO; } \\
\text { OLIVEIRA } / 2015 \\
\text { LILACS }\end{array}$ & $\begin{array}{l}\text { Diagnósticos de Enfermagem mais } \\
\text { frequentes em uma Unidade de } \\
\text { Terapia Intensiva Pediátrica }\end{array}$ & $\begin{array}{l}\text { Delineamento } \\
\text { Transversal }\end{array}$ & $\begin{array}{c}\text { Identificar os dez diagnósticos de enfermagem mais } \\
\text { frequentes em pacientes internados em uma Unidade de } \\
\text { Terapia Intensiva Pediátrica }\end{array}$ \\
\hline
\end{tabular}

FONTE: Dados da pesquisa.

Da análise foi possível identificar 91 diagnósticos de enfermagem definidos pelo enfermeiro, após julgamento clínico e uso do pensamento crítico, em pacientes internados na UTI de distintos serviços. Na tabela 01 estão os diagnósticos de enfermagem que alcançaram o IC $\geq 0,60$ conforme definido na metodologia como o ponto de corte para a análise da prevalência dos diagnósticos nos estudos que compuseram a amostra.

Tabela 01: Distribuição dos diagnósticos de enfermagem segundo sua prevalência nos estudos analisados. Senhor do Bonfim, BA, Brasil, 2016.

\section{DIAGNÓSTICOS DE ENFERMAGEM}

\section{ÍNDICE DE CONCORDÂNCIA}

(IC)

\begin{tabular}{lc}
\hline Risco de Infecção & 1,00 \\
Risco de Integridade da pele prejudicada & 0,72 \\
\hline Integridade da pele prejudicada & 0,72 \\
Risco de aspiração & 0,72 \\
Volume de líquido excessivo & 0,72 \\
Débito Cardíaco diminuído & 0,63 \\
Déficit de autocuidado para higiene íntima & 0,63 \\
Desobstrução Ineficaz das Vias aéreas & 0,63 \\
\hline Mobilidade física prejudicada & 0,63 \\
\hline Padrão Respiratório Ineficaz & 0,63 \\
\hline
\end{tabular}

FONTE: Dados das pesquisas. 


\section{DISCUSSÃO}

Foi possível evidenciar que houve uma predominância de estudos de caráter exploratório-descritivo publicados no período entre 2008 a 2015 , conforme apresentados no Quadro 01. Quanto ao uso dos sistemas de classificação, os estudos utilizaram predominantemente a Taxonomia da NANDA-I, apenas um estudo ${ }^{10}$ trabalhou com a CIPE®.

Após tratamento estatístico com a aplicação do IC foi evidenciado uma prevalência de 10 diagnósticos de enfermagem que atingiram o Índice de Concordância $\geq 0,60$ (Tabela 01). Estes estão distribuídos entre três dos 13 domínios da Taxonomia II da NANDA-I, sendo eles: Atividade/Repouso (cinco diagnósticos de enfermagem); Segurança/ Proteção (quatro diagnósticos de enfermagem); e Nutrição (um diagnóstico de enfermagem) ${ }^{11}$. Os demais domínios não apresentaram diagnósticos de enfermagem considerando a prevalência.

Dos 10 diagnósticos de enfermagem mais prevalentes, sete são diagnósticos reais e três de risco. Os diagnósticos reais correspondem às respostas humanas a uma condição de saúde (física, psicológica, social e/ou espiritual) e se caracterizam por presença de sinais e sintomas evidentes. Já os de riscos são situações onde se identificam uma vulnerabilidade diante um problema que deve ser evitado no planejamento da assistência de enfermagem ${ }^{11}$.

Considerando os diagnósticos de enfermagem relacionados à integridade tissular, a NANDA-I apresenta um diagnóstico de risco - "risco de integridade tissular prejudicada" definido como a vulnerabilidade a dano ao tecido e, outro diagnóstico real "integridade tissular prejudicada" definido como a presença do dano ao tecido (mucosa, córnea, tecido tegumentar, fáscia muscular, tendão, osso, cartilagem, cápsula articular e/ou ligamento) e caracterizado por tecido destruído ou lesado"1.

Os estudos apontaram um IC 0,72 relacionados a dois diagnósticos de enfermagem referentes à condição tissular, sendo um diagnóstico de risco e outro real, a saber: "risco de integridade da pele prejudicada" e "integridade da pele prejudicada" respectivamente. Quando se referem ao "risco de integridade da pele prejudicada", os estudos apontam fatores de riscos que predispõe às lesões cutâneas, ainda ausentes. São exemplos desses fatores de risco: pacientes acamados, com imobilização física (contenção), sedação contínua, sobrepeso/obesos, com retorno venoso lento, com dispositivos e evacuações frequentes; fatores que favorecem as possíveis lesões por pressão. $O$ diagnóstico real "integridade da pele prejudicada" refere-se a pacientes que apresentam comprometimento de pele/tecidos, como lesão por pressão, incisão/feridas cirúrgicas e/ou trauma ${ }^{12-14}$.

Ainda relacionada à integridade da pele, além dos diagnósticos já discutidos, há também a "mobilização física prejudicada" (IC 0,63$)$, o qual corresponde à limitação na capacidade de executar movimentos físicos independentes, intensificando o risco de lesões cutâneas e favorecendo o surgimento de lesão por pressão ${ }^{12}$. Como fatores etiológicos que direcionam ao diagnóstico de "mobilidade física prejudicada", foram evidenciados: vulnerabilidade hemodinâmica, culminando com a necessidade do uso de drenos, sondas, monitores e intubação; danos e disfunções neuromúsculo-esqueléticas; e a necessidade de restrição ao leito, devido às situações de agitação ou repouso significativo ${ }^{12-13}$.

A identificação destes diagnósticos se torna relevante por possibilitar o planejamento de ações preventivas, tais como: mudança de decúbito a cada duas horas, medidas para a diminuição de pressão em proeminências ósseas (colchões que aliviem a pressão, manutenção de roupas de cama esticadas), higiene, nutrição balanceada e avaliação diária da pele ${ }^{12}$.

A higiene pessoal também é condição que assegura a integridade da pele, sendo identificada a prevalência de dois diagnósticos: "déficit de autocuidado para banho/higiene" e "déficit de autocuidado para higiene íntima", contudo apenas o segundo alcançou o IC $\geq 0,60$.

A clientela da terapia intensiva é composta por pacientes que têm dificuldades para executar as atividades de higiene pessoal em razão do uso de dispositivos como sondas e cateteres, da dificuldade de locomoção e até mesmo da dor, requerendo a atenção da equipe de enfermagem frente às 
necessidades básicas de higiene e alimentação ${ }^{12}$.

A nutrição também é um fator importante frente à manutenção da pele íntegra, sendo identificado um diagnóstico com IC 0,72 - "volume de líquido excessivo" definido na NANDA-I" como retenção aumentada de líquidos isotônicos. Por se tratar de pacientes hemodinamicamente instáveis, os profissionais de enfermagem devem estar atentos às necessidades básicas de higiene e alimentação ${ }^{13}$.

Os cuidados com a pele são relevantes, pois qualquer dano à integridade da pele pode ocasionar falhas na primeira linha de defesa do organismo, potencializando o "risco de infecção", o que além de ser uma característica definidora é também considerado um diagnóstico de enfermagem ${ }^{14}$. E como diagnóstico, ○ "risco de infecção" foi evidenciado em todos os estudos que compuseram a amostra.

Tal prevalência demonstra a vulnerabilidade dos pacientes frente aos microrganismos patogênicos, relacionado exatamente à condição crítica do paciente, visto que esse dispõe de fatores de risco como procedimentos invasivos, usos de diversos dispositivos e o próprio comprometimento imunológico peculiar a uma pessoa em estado de adoecimento e na UTI. Tais fatores de risco são bem detalhados nos estudos, sendo eles: procedimentos invasivos, feridas cirúrgicas, comorbidades, doenças crônicas, estado nutricional prejudicado, emprego de terapias imunossupressoras (agentes farmacêuticos) e a própria defesa primária e secundáriaa ${ }^{1214-15}$.

Em relação ao comprometimento do sistema cardiopulmonar, também forma evidenciados diagnósticos de risco e reais, como: "risco de aspiração", "débito cardíaco diminuído", "padrão respiratório ineficaz" e "desobstrução ineficaz das vias aéreas". Embora tenha sido identificado apenas um estudo direcionado a UTI cardiológica, o diagnóstico de "débito cardíaco diminuído" está presente em mais da metade dos estudos analisados (IC 0,63).

Entre os diagnósticos associados ao aparelho respiratório foram evidenciados três diagnósticos. O diagnóstico de enfermagem "risco de aspiração" está associado ao fato de que os pacientes na UTI costumam estarem entubados, muitos sob sedação, inconscientes e alimentando-se por sonda nasoenteral ou nasogástrica, com mobilidade intestinal diminuída. Estes são fatores de risco à entrada de secreções gastrointestinais ou orofaríngeas (sólidas ou líquidas) na árvore traqueobrônquica ${ }^{13}$. Esses fatores de risco dão sustentação ao diagnóstico de enfermagem "desobstrução ineficaz de vias aéreas", diante da incapacidade de eliminar secreções e do risco em provocar obstruções, comprometendo a manutenção de uma via aérea pérvia ${ }^{14}$.

Um diagnóstico relevante e relacionado ao aparelho respiratório, mas que não alcançou o IC foi a "troca de gases prejudicada" (IC 0,54). Esse diagnóstico foi associado aos resultados alterados no exame de gasometria e pH sanguíneo, confirmando alterações na concentração dos gases oxigênio e dióxido de carbono $^{13}$. Uma possível explicação para o não alcance do IC para este diagnóstico pode estar associada a classificado do diagnóstico no domínio "atividade/repouso", ao tempo em que "padrão respiratório ineficaz" e "desobstrução ineficaz das vias áreas" estão classificados como pertencentes ao domínio "eliminação/troca".

Com a decisão clínica pelos diagnósticos de "padrão respiratório ineficaz" e "desobstrução ineficaz das vias áreas", surgem os cuidados a intervir nos fatores etiológicos destes diagnósticos: confirmar o posicionamento e aspirar sonda gástrica antes de infundir a dieta; observar presença de distensão abdominal e estase gástrica; examinar o abdome; manter $\circ$ paciente em fowler por um período de duas horas após a infusão da dieta; e observar a ocorrência de eliminações intestinais².

No decorrer dos levantamentos dos diagnósticos de enfermagem percebeu-se que alguns diagnósticos não alcançaram ○ IC $\geq 0,60$ por existir uma variação na redação dos títulos. Alguns deles são escritos com um título mais abrangente e outros, mais específico, comprometendo a uniformização da linguagem terminológica na descrição fidedigna dos diagnósticos de enfermagem.

Como exemplo dessa problemática pode ser citado o diagnóstico de enfermagem "dor" que embora não tenha alcance o IC $\geq 0,60$, é um diagnóstico relevante e bastante presente no contexto da terapia intensiva, associado às características definidoras como: inquietação, agitação, angústia e 
irritabilidade pela ameaça ou mudança no estado de saúde e de papel social. Condições comuns nos pacientes internados na UTI ${ }^{16}$. Esse diagnóstico apresenta variação no título, de modo que em uns estudos aparece apenas como "dor"12-13 enquanto em outros está descrito de modo específico conforme consta na Taxonomia da NANDA-I: "dor aguda"2,10,14,17-19 e "dor crônica"19. O diagnóstico com foco na dor aparece em sete dos estudos analisados, contudo com essa variação na definição do título entre "dor", "dor aguda" e "dor crônica".

A mesma variação ocorre nos diagnósticos referentes ao autocuidado. Num estudo ${ }^{12}$ apresenta de modo generalizado o problema em relação ao autocuidados decidindo pelo diagnóstico de "déficit de autocuidado", já em outro estudo ${ }^{10}$ apresenta com especificação para qual o déficit de autocuidado, a saber: "déficit de autocuidado para higiene íntima"; "déficit de autocuidado para banho/higiene" e "déficit de autocuidado para alimentação". Uma possível justificativa do porquê o "déficit de autocuidado para higiene íntima" ter alcançado o IC $\geq 0,60$ e o diagnóstico "déficit de autocuidado para banho/higiene" não. O diagnóstico "déficit de autocuidado" também aparece em sete dos estudos analisados, com variações como autocuidado para: banho/higiene, higiene íntima, para vestir-se e para alimentação.

Observa-se também essa situação nas apresentações dos títulos dos diagnósticos em relação à termorregulação. Na NANDA-I ${ }^{11}$ constam os seguintes diagnósticos: "risco de desequilíbrio de temperatura corporal", "termorregulação ineficaz", "hipertermia" e "hipotermia". Nos estudos foram identificados estes títulos e mais um que foi o "risco para hipotermia" 12 , o qual não constava nas edições anteriores da NANDA-I e sendo incluído apenas na edição de $2015^{11}$.

Por fim, dentre os diagnósticos com maior variação em relação ao seu título, apesar dos estudos adotarem o sistema de classificação da NANDA-I, vale ressaltar o diagnóstico relacionado à "perfusão tissular", o qual aparece como: "risco de perfusão tissular periférica ineficaz"10,20; "perfusão tissular alterada"13,15; "perfusão periférica ineficaz"2,17-19; "perfusão tissular ineficaz: cerebral",12,15,18; "perfusão tissular ineficaz: cardiopulmonar", 15,18,20; "risco de perfusão tissular cardíaca diminuída"17; "perfusão tissular gastrointestinal" ${ }^{2,18}$; "risco de perfusão renal ineficaz"17,20 e "perfusão tissular ineficaz: renal" ${ }^{\prime 2,18-19}$.

Assim, evidencia-se que a variabilidade entre a definição do título do diagnóstico acaba por interferir na identificação da prevalência em função das respostas humanas afetadas, tornando alguns diagnósticos menos prevalentes em razão das variações na descrição dos seus títulos. Fato que reforça a necessidade de uma linguagem padronizada para o registro dos problemas de enfermagem.

\section{CONCLUSÕES}

O presente estudo identificou dez diagnósticos de enfermagem como mais prevalentes diante do julgamento clínico do enfermeiro na assistência ao paciente na UTI. Mediante o levantamento desses diagnósticos foi possível evidenciar que na UTI são mais prevalentes os diagnósticos reais do que os de riscos.

Outro achado que vale considerar é a redação dos títulos diagnósticos, que em alguns estudos é feita com uma abrangência geral e em outros, mais específica, o que pode comprometer a uniformização da linguagem terminológica na descrição fidedigna dos diagnósticos de enfermagem comprometendo $\circ$ planejamento dos cuidados.

Com a identificação dos diagnósticos mais prevalentes no contexto da UTI é possível traçar o perfil clínico e epidemiológico das demandas dos pacientes internados, favorecendo a uma melhor assistência, baseada na capacitação/educação permanente e num cuidado mais efetivo e de qualidade. 


\section{REFERÊNCIAS}

1. Paes GO, Mesquita MGR, Moreira SO, Monteiro $A B F$. Fundamentação das práticas de cuidar frente ao paciente crítico. In: Silva RS, Bittencourt IS, Paixão GPN. Enfermagem avançada: um guia para a prática. Salvador: SANAR; 2016. P. $467-$ 85.

2. Truppel TC, Meler MJ, Calixto RC, Peruzzo AS, Crozeta K. Sistematização de enfermagem na Unidade de Terapia Intensiva. Rev Bras Enferm. 2009;62(2):221-227. doi: 10.1590/S003471672009000200008

3. Adams F, Rodrigues FCP, Fontana RT. The light technologies in the nursing assistance in the units of intensive therapy. Rev enferm UFPE on line. $2011 ; 5(2): 417-25$.

4. COFEN. Conselho Federal de Enfermagem (RJ). Resolução COFEN N. 358/2009, de 15 de outubro de 2009: Sistematização da Assistência de Enfermagem e a implementação do Processo de Enfermagem em ambientes, públicos ou privados, em que ocorre o cuidado profissional de Enfermagem. Brasília: COFEN; 2009.

5. Pereira A, Silva RS. O corpo em situações clínicas: processo de cuidar na Enfermagem. In: Figueiredo NMA, Machado WCA. Tratado Cuidados de Enfermagem Médico-Cirúrgico. V. 1. Cap. 45. São Paulo: Roca; 201 2. P. 705-29.

6. Tannure CM, Pinheiro AM. Sistematização da Assistência de Enfermagem: Guia Prático. 2. ed. Rio de Janeiro: Guanabara Koogan; 2010.

\section{Mendes KDS, Silveira RCCP, Galvão CM.} Revisão Integrativa: método de pesquisa para a incorporação de evidências na saúde e na enfermagem. Rev. Texto Contexto-Enferm. 2008;17(4)758-64. doi: 10.1590/s010407072008000400018

8. Souza MT, Silva MD, Carvalho R. Revisão Integrativa: o que é e como fazer. Rev. Einstein. 2010;8(1)102-6.

9. COFEN. Conselho Federal de Enfermagem
(RJ). Resolução COFEN n.272, de 27 de agosto de 2002: Sistematização da Assistência de Enfermagem (SAE) nas instituições de Saúde. Rio de Janeiro: COFEN; 2002.

10. Cruz APO, Lopes R. Diagnóstico de enfermagem no pós-operatório de cirurgias cardíacas. Rev. Salusvita. 2010;29(3):293-312.

11. Herdman TH. Diagnósticos de enfermagem da NANDA: definições e classificação - 2015/2017. Porto Alegre: Artmed; 2015.

12. Prado $P R$, Beccaria $L M$, Contrin LM. Principais diagnósticos de enfermagem em unidade de terapia intensiva. CuidArte, Enferm. 2009;3(2):176-83.

13. Salomé GM. Diagnóstico de enfermagem dos pacientes internados em uma Unidade de Terapia Intensiva. Rev. Saúde Coletiva. 2011 ;8(47):24-8.

14. Guedes DMB, Rossato LM, Oliveira EA. Diagnósticos de enfermagem mais frequentes em uma unidade de terapia intensiva pediátrica. Rev Enferm UFSM. 2015;5(3):476-85. doi: $10.5902 / 2179769216471$

15. Carvalho EC, Martins FTM, Dalri MCB, Canini SRMS, Laus AM, Bachion MM et al. Relações entre a coleta de dados, diagnósticos e prescrições de enfermagem a pacientes adultos de uma unidade de terapia intensiva. Rev. Latino-Am. Enfermagem. 2008; 16(4):700-6. doi: 10.1590/S010411692008000400008

16. Oliveira MF, Freitas MC. Diagnósticos e intervenções de enfermagem frequentes em mulheres internadas em unidade de terapia intensiva. Rev Bras Enferm. 2009;62(3):343-8. doi: $10.1590 /$ S0034-71672009000300002

17. Almeida DV, Oliveira KF, Oliveira JF, Pires NF, Filgueira VSA. Diagnósticos de enfermagem mais frequentes em pacientes internados na Unidade de Terapia Intensiva Coronariana. Arq Med Hosp Fac Cienc Med Santa Casa São Paulo. 2013;58(2):649.

18. Paganin A, Menegat $P$, Klafke T, Lazzarotto $A$, Fachinelli TS, Chaves IC, Souza EN. Implantação 
do diagnóstico de enfermagem em unidade de terapia intensiva: uma análise periódica. Rev Gaúcha Enferm. 2010;31(2):307-313. doi: $10.1590 /$ S1983-14472010000200015

19. Chianca TCM, Lima APS, Salgado PO.

Diagnósticos de enfermagem identificados em pacientes internados na unidade de terapia intensiva adulto. Rev Esc Enferm USP. 2012 ;46(5): $1102-8$. doi: 10.1590/S008062342012000500010

20. Ramos GS, Santana LC, Ferreira PHC, Chianca TCM, Guedes HM. Diagnósticos de enfermagem documentados em prontuários de pacientes em unidade de terapia intensiva. Rev. enferm. Cent.Oeste Min. 2013;3(2):679-86. doi: 10.19175/ recom.v0i0.382 